\title{
A Semi-Lagrangian Approach For American Asian Options Under Jump Diffusion
}

\author{
Y. d’Halluin*, P.A. Forsyth ${ }^{\dagger}$, and G. Labahn ${ }^{\ddagger}$ \\ December 15, 2003 \\ version 1.7
}

\begin{abstract}
A semi-Lagrangian method is presented to price continuously observed fixed strike Asian options. At each timestep a set of one dimensional partial integral differential equations (PIDEs) is solved and the solution of each PIDE is updated using semi-Lagrangian timestepping. Crank-Nicolson and second order backward differencing timestepping schemes are studied. Monotonicity and stability results are derived. With low volatility values, it is observed that the non-smoothness at the strike in the payoff affects the convergence rate; sub-quadratic convergence rate is observed.
\end{abstract}

Keywords: Continuously observed Asian option, semi-Lagrangian, American option, jump diffusion, implicit discretization.

Acknowledgment: This work was supported by the Natural Sciences and Engineering Research Council of Canada, the Social Sciences and Humanities Research Council of Canada and RBC Financial Group.

\section{Introduction}

An Asian option gives the holder a payoff that depends on the average price of the underlying asset over a specified period of time. Asian-style derivatives have a wide variety of applications in equity, energy, interest rate, and insurance markets. To the best of our knowledge, they were first introduced in [11]. For an historical review of Asian options, see [10].

Asian derivatives are very popular for several economic reasons. First, since the volatility of the average price is less than the price itself, Asian options are less expensive than regular vanilla options. Second, while for some classes of derivatives it is possible for large market participants to manipulate the price of illiquid commodities, it is much harder to manipulate the average price over a period of time. Finally, companies are often more interested in the average price of oil or foreign exchange rate, than the underlying price or rate at a specific date when considering long term projects. For example, airline companies are certainly more interested in buying oil based on its average price instead of its spot price. Consequently, pricing Asian options accurately is critical.

The price of an Asian option at any time is a function of both the underlying asset at that time and the average of the underlying prices up to that time; as such these options are considered path-dependent.

\footnotetext{
*School of Computer Science, University of Waterloo, Waterloo ON, Canada N2L 3G1 (e-mail: ydhallui@elora.uwaterloo.ca).

${ }^{\dagger}$ School of Computer Science, University of Waterloo, Waterloo ON, Canada N2L 3G1 (e-mail: paforsyt@elora.uwaterloo.ca).

$\ddagger$ School of Computer Science, University of Waterloo, Waterloo ON, Canada N2L 3G1 (e-mail:glabahn@scg.uwaterloo.ca)
} 
In practice, Asian option contracts typically specify that the average is monitored discretely. A typical situation would be to base the average on the daily closing price. If daily averaging is used, then for typical market parameters, for options with expiry times more than three months, we can consider these options as being continuously monitored, for all practical purposes. In addition, if we need to price long term (greater than one year) Asian options, then using timesteps of one day (which would be required in a discrete observation model [42]) would clearly be computationally wasteful. Consequently, in this paper we focus on continuously observed Asian options. For details on numerical methods for discretely observed Asian options, we refer the reader to [15, 14, 42, 22]

In general, a two dimensional PDE must be solved to determine the price of an Asian option. In certain special cases, for example, constant volatility, no barrier features, and a floating strike contract, this problem can be reduced to a one-dimensional PDE [4]. In addition, for either floating or fixed strike, but not American style or asset dependent features (e.g. volatility a function of asset price), a one dimensional PDE can be derived [38].

However, in the general case (that is, American style, asset dependent barriers or volatility), the two dimensional PDE cannot be reduced to one dimension. This PDE is difficult to solve, since the problem has no diffusion in one of the coordinate directions. In [41, 44], a flux limiter was used to retain accuracy while preventing oscillations. In [27], the first order hyperbolic term was discretized using a first order upwind type method, resulting in at most first order accuracy. In [30], a semi-Lagrangian method was used to discretize the hyperbolic term in the average direction. Semi-Lagrangian schemes were first introduced by [18] and [32] for atmospheric and weather numerical predictions. These are time marching schemes that integrate convection-diffusion equations by tracing backward in time the position of the flow. These schemes are used to reduce numerical problems raised by convection dominated equations. In principle, provided an appropriate time discretization is used, and a high enough order of interpolation is used to recover values at the feet of the characteristic curves [20,1,9], then this method is capable of greater than first order convergence as the grid and timestep size is reduced.

There is increasing empirical evidence that the usual assumption of geometric Brownian motion should be augmented by discontinuous jump processes[19]. Such models were originally introduced in the option valuation context in [28]. It is also possible to develop more complex valuation models which include stochastic volatility as well as jumps [7].

In [3], the authors have suggested that good results can be obtained in practice by combining the deterministic volatility approach with lognormally distributed Poisson jumps with constant parameters. They argue that this avoids most of the difficulties associated with calibrated local volatility surfaces, while retaining the simplicity of a single factor model, which is an important practical consideration.

In this paper, we will explore the use of a semi-Lagrangian method for pricing American Asian options with jump diffusion. As we will make no assumptions regarding the contract, or the form of the volatility function, this will result, in the European case, in a two dimensional Partial Differential Integral Differential Equation (PIDE). In the American case, the problem becomes a partial differential integral linear complementarity problem. After semi-discretization in time, the semi-Lagrangian approach results in a set of one-dimensional differential integral inequalities. At each timestep, these one dimensional problems are independent. Hence the methods in $[16,17]$ can be used to solve each discrete problem. We remark that although we focus exclusively on Asian options in this paper, similar PDEs (no diffusion in one of the space-like directions) occur in certain interest rate models [36], and hence the methods developed here will be applicable to this case as well.

The main results in this paper are

- We demonstrate that a semi-Lagrangian method can be used to price continuously observed American Asian options under jump diffusion processes. The implementation suggested here reduces this problem to solving a decoupled set of one dimensional nonlinear discrete partial integral differential 
equations (PIDEs) at each timestep. This makes implementation of this method very straightforward in a software library which is capable of pricing discretely observed path dependent options [42].

- We show that in the fully implicit case, the semi-Lagrangian method is algebraically identical to a standard numerical method for pricing discretely observed Asian options, if the observation interval is equal to the discrete timestep. Since lattice methods [39] can be regarded as explicit finite difference methods it follows that the usual binomial forest method for Asian options [24] can also be regarded as an explicit semi-Lagrangian method.

- Since the discretized problem at each timestep reduces to a set of decoupled one dimensional PIDEs, we can make use of the techniques developed in $[21,16,17]$ to prove certain properties of the discrete scheme, including convergence of the iterative method used to solve the implicit discrete equations. In the fully implicit case, it is straightforward to prove $l_{\infty}$ stability and monotonicity, which are important properties of discrete schemes for option pricing $[5,34,13,12]$.

- We include experimental computations which indicate that, even if second order timestepping methods are used, observed convergence as the mesh and timestep is refined occurs at a sub-second order rate. The problem can be traced to the non-smoothness of the payoff function.

The remainder of the paper is organized as follows. In the next section we give the PDE formulation for the American Asian options problem under jump diffusion. In Section 3 we show how this can be viewed as a semi-Lagrangian problem while the following section gives a relationship between the semi-Lagrangian problem and the case of the standard method for pricing discrete Asian options. Sections 5 and 6 includes a number of important properties of our discretization. Section 7 presents the iterative algorithm along with its convergence properties. Numerical results appear in section 8 with the last section giving our conclusions and topics for future research.

\section{Mathematical Model}

In this section we give the mathematical model for options with jump diffusion processes. We do this for both European and American options. If the underlying asset follows a jump diffusion process, the usual portfolio hedging arguments cannot be used. We will also present a brief discussion of various strategies for hedging jump risk.

Let $S$ represent the underlying stock price. Then potential stock paths followed by the stock can be modeled by a stochastic differential equation given by

$$
\frac{d S}{S}=(\xi-\kappa \lambda) d t+\sigma d Z+(\eta-1) d q
$$

where

$$
\begin{aligned}
\xi & \text { is the drift rate, } \\
d q & \text { is the independent Poisson process, }= \begin{cases}0 & \text { with probability } 1-\lambda d t \\
1 & \text { with probability } \lambda d t,\end{cases} \\
\lambda & \text { is the mean arrival time of the Poisson process, } \\
\eta-1 & \text { is an impulse function producing a jump from } S \text { to } S \eta, \\
\sigma & \text { is the volatility, } \\
d Z & \text { is an increment of the standard Gauss-Wiener process, } \\
\kappa & \text { is } E[\eta-1], \text { where } \\
E[\cdot] & \text { is the expectation operator. }
\end{aligned}
$$


When the average is monitored continuously $[6,39,22]$, the arithmetic average $A$ is defined as

$$
\begin{aligned}
A & =\frac{\int_{0}^{t} S(u) d u}{t}, \\
d A & =\frac{(S-A) d t}{t} .
\end{aligned}
$$

\subsection{The PDE for Asian Options}

Using standard arguments, [6] the value of an option depending on $A$ (2.2) and $S$ (2.1) is given by (assuming no jumps, that is, $\lambda=0$ )

$$
V_{t}+\frac{1}{2} \sigma^{2} S^{2} V_{S S}+\frac{(S-A)}{t} V_{A}+r S V_{S}-r V=0
$$

where $r$ is the continuously compounded risk free interest rate. Since we are solving backward in time from the expiration time $t=T$ to the present time $t=0$, equation (2.3) becomes

$$
V_{\tau}=\frac{1}{2} \sigma^{2} S^{2} V_{S S}+\frac{(S-A)}{T-\tau} V_{A}+r S V_{S}-r V
$$

where $\tau=T-t$. It is important to note that equation (2.4) has no diffusion term in the $A$ direction and this is the source of many numerical difficulties [41].

Extending equation (2.4) to the case of jumps gives

$$
V_{\tau}=\frac{(S-A)}{T-\tau} V_{A}+\frac{1}{2} \sigma^{2} S^{2} V_{S S}+(r-\lambda \kappa) S V_{S}-r V+\left(\lambda \int_{0}^{\infty} V(S \eta) g(\eta) d \eta-\lambda V\right),
$$

where

$g(\eta) \quad$ is the probability density function of the jump amplitude $\eta$,

$$
\text { thus for all } \eta: g(\eta) \geq 0 \text { and } \int_{0}^{\infty} g(\eta) d \eta=1 \text {. }
$$

As a specific example, consider the probability density function suggested by [28, 37]:

$$
g(\eta)=\frac{e^{\left(-\frac{(\log (\eta)-\mu)^{2}}{2 \gamma^{2}}\right)}}{\sqrt{2 \pi} \gamma \eta}
$$

If $E[\cdot]$ is the expectation operator,

$$
E[\eta]=\int_{0}^{\infty} \eta g(\eta) d \eta
$$

then, $E[\eta]=\exp \left(\mu+\gamma^{2} / 2\right)$, which means that the expected relative change in the stock price is given by $\kappa=E[\eta-1]=\exp \left(\mu+\gamma^{2} / 2\right)-1$. For brevity, the details of the derivation of equation (2.5) have been omitted (see [3, 28, 39]). In general, it is not possible to construct a hedging portfolio which eliminates jump risk. However, by adding options to the hedging portfolio, a hedging strategy can be constructed which minimizes jump risk [3]. If equation (2.5) is calibrated to market prices, then the parameters so obtained should be regarded as risk-adjusted [26], not historical.

If we define

$$
\mathcal{H} V \equiv V_{\tau}-\left(\frac{S-A}{T-\tau} V_{A}+\frac{\sigma^{2} S^{2}}{2} V_{S S}+(r-\lambda \kappa) S V_{S}-(r+\lambda) V+\lambda \int_{0}^{\infty} V(S \eta) g(\eta) d \eta\right)
$$

and if $V^{*}(S, A)$ is the payoff, then the American option pricing problem can be stated as [31]

$$
\min \left(\mathcal{H} V ; V-V^{*}\right)=0 .
$$




\subsection{Boundary Conditions for our PIDE}

In order to completely specify our problem we still need to give boundary conditions for our American Asian option pricing PIDE.

For the terminal boundary conditions, a number of common payoffs for pricing different types of Asian securities can be used. Typical examples include

- fixed strike call: $V(S, A, \tau=0)=\max (A-K, 0.0)$,

- fixed strike put: $V(S, A, \tau=0)=\max (K-A, 0.0)$.

For the non-terminal boundary conditions, note first that at $S=0$ we have that $\mathcal{H} V$ reduces to

$$
\mathcal{H} V=V_{\tau}-\left(\frac{-A}{T-\tau} V_{A}+r V\right) ; S \rightarrow 0 .
$$

The boundary condition at $S \rightarrow \infty$ is, however, more difficult to specify and requires additional justification. If we make the common assumption that $V_{S S} \rightarrow 0$ as $S \rightarrow \infty$, then this implies that

$$
V \simeq f(A, \tau) S+g(A, \tau)
$$

as $S \rightarrow \infty$ which then means that equation (2.9) becomes

$$
\mathcal{H} V \simeq V_{\tau}-\left(\frac{S-A}{T-\tau} V_{A}+r S V_{S}-r V\right) ; S \rightarrow \infty
$$

For $A \gg K$ and $S \rightarrow \infty$, we can approximate the solution to $\mathcal{H} V=0$ by

$$
V \simeq H_{1}(\tau) A+H_{2}(\tau) S+H_{3}(\tau)
$$

so that

$$
V \simeq \frac{D_{1}}{T} e^{-r \tau}(T-\tau) A+\left[\frac{D_{1}}{r T}\left(1-e^{-r \tau}\right)+D_{2}\right] S+D_{3} e^{-r \tau}
$$

where $D_{1}, D_{2}, D_{3}$ are independent of $(S, A, \tau)$ and are determined by the payoff. For example, for a fixed strike call, $D_{1}=1, D_{2}=0, D_{3}=-K$. We then use equation (2.15) at all points on $S=S_{\max }$ (which is clearly an approximation for $A$ small) so that

$$
V_{S} \simeq\left[\frac{D_{1}}{r T}\left(1-e^{-r \tau}\right)+D_{2}\right] ; S \rightarrow \infty .
$$

Substituting equation (2.16) into equation (2.13) gives

$$
\mathcal{H} V \equiv V_{\tau}-\left(\frac{S-A}{T-\tau} V_{A}+\chi(S, \tau)-r V\right) ; S \rightarrow \infty
$$

where

$$
\chi(S, \tau)=\left[\frac{D_{1}}{r T}\left(1-e^{-r \tau}\right)+D_{2}\right] r S .
$$

The use of approximation (2.16) is discussed in [29], where it is mentioned that estimate (2.16) is in fact an upper bound for $V_{S}$. It must be admitted that use of equation (2.15) at all points along $S=S_{\max }$ is not 
rigorously justified. However, we note that other authors [27] simply specify that the boundary condition at $S=S_{\max }$ is set to the payoff. In [27], the size of the computational domain is increased as the grid size is reduced, so that the effect of poor specification of the boundary condition becomes negligible. However, as discussed in [29], the boundary condition (2.17) is at least qualitatively correct.

Note that no boundary conditions are required at $A=0$ or as $A \rightarrow \infty$, since the characteristics of the PIDE are outgoing in the $A$ direction. In practice, we solve for the solution of equation (2.10) on the finite computational domain $\left[0, S_{\max }\right] \times\left[0, A_{\max }\right]$. Usually $S_{\max }=A_{\max }$. If $S_{\max }$ is sufficiently large, then the errors introduced by imposing condition (2.17) are small in regions of interest. This will be verified in some numerical experiments.

For the payoffs mentioned earlier, the corresponding formulae for $\chi(\tau, S)$ are given by

- fixed strike call: $\chi(\tau, S)=\frac{\left(1-e^{-r \tau}\right)}{T} S$

- fixed strike put: $\chi(\tau, S)=0$.

\subsection{Additional Observations}

In general, there may be no smooth solutions to equation (2.10). In what follows it will be understood that we are seeking weak viscosity solutions to equation (2.10). A detailed discussion concerning existence and uniqueness of viscosity solutions to equation (2.10), can be found in [31,2], for European and American options under jump diffusion. In addition, sufficient conditions to ensure convergence of a discrete numerical scheme to the viscosity solution, is given in [5]. Finally, an application of the results in [5] to the case of European options with jump diffusion is given in $[12,13]$.

Typically when pricing continuously observed arithmetic average Asian option, a two dimensional problem must be solved (2.4). In [41], the authors use a finite volume approach combined with flux limiters to solve equation (2.4). This requires solution of a set of nonlinear discretized algebraic equations at each timestep. When the convection terms becomes very large, (note that the convection term in the $A$ direction in equation (2.5) becomes infinite as $\tau \rightarrow T$ ), flux-limiters revert to a first order upwind scheme which affects the accuracy of the solution [1]. In this paper, we suggest an alternative approach to solve equation (2.4) using a semi-Lagrangian scheme. This idea was also suggested for American Asian options (without jumps but with stochastic volatility) in [30].

\section{Semi-Lagrangian Discretization}

This section explores different discretization methods for the partial differential equation using the semiLagrangian approach. Before proceeding any further, let us introduce the following definitions. We use an unequally spaced grid in $S$ coordinates for the PDE discretization $\left[S_{0}, \ldots, S_{M}\right]$, and similarly use an unequally spaced grid in the $A$ direction $\left[A_{0}, \ldots A_{M}\right]$. Let

$$
V_{i, j}^{n}=V\left(S_{i}, A_{j}, \tau^{n}\right)
$$

denote the solution at asset price node $S_{i}$ for the average $A_{j}$ and time level $n$. Let $C$ be the differential operator represented by

$$
C V=\frac{1}{2} \sigma^{2} S^{2} V_{S S}+(r S-\lambda \kappa) V_{S}-(r-\lambda) V
$$

and

$$
\mathcal{B} V=\lambda \int_{0}^{\infty} V(S \eta) g(\eta) d \eta
$$


Equation (2.5) can then be rewritten as

$$
V_{\tau}+\frac{(A-S)}{T-\tau} V_{A}=C V+\mathcal{B} V
$$

We will use a penalty method $[16,21,43]$ to impose the American early exercise constraint. Briefly, this replaces equation 2.10 by a non-linear PIDE

$$
V_{\tau}+\frac{(A-S)}{T-\tau} V_{A}=C V+\mathcal{B} V+q(V)
$$

where the penalty term is such that

$$
q(V)=\rho \max \left(V^{*}-V, 0\right) .
$$

The penalty parameter $\rho$ is selected so that

$$
\left|V-V^{*}\right|<\text { tol when } V<V^{*}
$$

where $t o l$ is a user specified tolerance. It is easy to solve the discrete equations with tol less than the discretization error, so this error in determining the numerical solution to equation (2.10) is not of practical concern. We refer the reader to [21] for more details about this method.

We use standard finite difference methods to discretize the operator $C V$ [34] (see Appendix A). If we impose boundary condition (2.17), and use forward and backward differencing as appropriate, it is easy to see that the discrete form of $I-C V$ is an M-matrix (see Section 5.2). As discussed in [34], for typical values of $\sigma, r$, upwind differencing of the $V_{S}$ term in equation (3.5) is required only rarely, and usually remote from regions of interest, so that in practice this does not impact solution quality. Requiring the discrete form of $C V$ to be an M-matrix has interesting theoretical properties. In the following, we denote the discrete form of $C V$ at $S=S_{i}, A=A_{j}, \tau=\tau^{n}$ by $(C V)_{i, j}^{n}$. As described in [17, 16] the integral $(\mathcal{B} V)_{i, j}$ can be efficiently computed by transforming to equally spaced $\log S$ coordinates, approximating the integral using a Trapezoidal rule, using an FFT, and then transforming back to $S$ coordinates. Special care is taken to avoid problems with wrap around [17]. If linear interpolation is used to transform from equally spaced $\log S$ coordinates to unequally spaced $S$ coordinates (and vice versa), this introduces a second order error consistent with the discretization of the PDE terms [17]. Effectively, we are approximating $\mathcal{B V}$ by

$$
\begin{aligned}
(\mathcal{B} V)_{i, j} \simeq & \sum_{k} b_{i k} V_{k j} \\
= & B \cdot V_{j} \\
& 0 \leq b_{i k} \leq 1 \text { and } \sum_{k} b_{i k} \leq 1 .
\end{aligned}
$$

The dense matrix multiply $B \cdot V_{j}$ can be evaluated efficiently using an FFT. For details, see $[17,16]$.

The Lagrangian derivative along a trajectory $A=A(S, \tau)$, for $S$ fixed, is

$$
\frac{D V}{D \tau}=\frac{\partial V}{\partial \tau}+\frac{\partial V}{\partial A} \frac{d A}{d \tau} .
$$

Along the trajectory

$$
\frac{d A}{d \tau}=\frac{A-S}{T-\tau}
$$

equation (3.5) can be written as

$$
\frac{D V}{D \tau}=C V+\mathcal{B} V+q(V) .
$$


Let $A=A\left(S_{i}, A_{j}, \tau^{n+1}, \tau\right)$ along a trajectory satisfying equation (3.10), which passes through the discrete grid point $\left(S_{i}, A_{j}\right)$ at $\tau=\tau^{n+1}$ for $S_{i}$ being held constant. Let $A_{j(i, n+1)}^{n}$ be the departure point of this trajectory at $\tau=\tau^{n}$. Note that $A_{j(i, n+1)}^{n}$ will not necessarily coincide with a grid point $A_{j}$. Rather $A_{j(i, n+1)}^{n}$ is determined by solving

$$
\begin{aligned}
\frac{d A}{d \tau}= & \frac{A-S_{i}}{T-\tau} \\
& A=A_{j} ; \tau=\tau^{n+1},
\end{aligned}
$$

from $\tau=\tau^{n+1}$ to $\tau=\tau^{n}$, that is,

$$
A_{j(i, n+1)}^{n}=A_{j}^{n+1}+\int_{\tau^{n+1}}^{\tau^{n}} \frac{A-S_{i}}{T-\tau} d \tau
$$

Let $V_{i, j(i, n+1)}^{n}=V\left(S_{i}, A_{j(i, n+1)}^{n}, \tau^{n}\right)$ denote the value of the option price at the departure point of the trajectory. Then discretizing equation (3.11) along the characteristic trajectory for different timestepping schemes gives, in the case of fully implicit timestepping:

$$
\frac{V_{i, j}^{n+1}-V_{i, j(i, n+1)}^{n}}{\Delta \tau}=(C V)_{i, j}^{n+1}+(\mathcal{B} V)_{i, j}^{n+1}+q\left(V_{i, j}^{n+1}\right),
$$

and for Crank-Nicolson timestepping $(\mathrm{CN})$,

$$
\frac{V_{i, j}^{n+1}-V_{i, j(i, n+1)}^{n}}{\Delta \tau}=\frac{1}{2}\left((C V)_{i, j}^{n+1}+(\mathcal{B} V)_{i, j}^{n+1}\right)+\frac{1}{2}\left(\left(C V_{i, j(i, n+1)}\right)^{n}+(\mathcal{B} V)_{i, j(i, n+1)}^{n}\right)+q\left(V_{i, j}^{n+1}\right),
$$

and for second order backward differencing (BDF) [8]

$$
\frac{\frac{1}{2} V_{i, j}^{n+1}-2 V_{i, j(i, n+1)}^{n}+\frac{1}{2} V_{i, j(i, n+1)}^{n-1}}{\Delta \tau}=(C V)_{i, j}^{n+1}+(\mathcal{B} V)_{i, j}^{n+1}+q\left(V_{i, j}^{n+1}\right) .
$$

For ease of exposition, we have written equation (3.16) for constant timesteps. This is trivially generalized to non-constant timesteps [8].

Unlike traditional applications of the semi-Lagrangian approach where the characteristic curve must be estimated numerically, for Asian options the solution along the characteristic curve can be determined exactly. Regarding $S$ as a constant, and solving equation (3.10) gives

$$
A=S_{i}+\frac{D}{T-\tau}
$$

where $D$ is a constant independent of $A$ (but a function of $S_{i}$ ). At $\tau=\tau^{n+1}, A=A_{j}^{n+1}$, so that

$$
\begin{aligned}
\text { At time } \tau^{n}: & A_{j(i, n+1)}^{n}=A_{j}^{n+1}+\frac{\left(S_{i}-A_{j}^{n+1}\right)\left(\tau^{n+1}-\tau^{n}\right)}{T-\tau^{n}}, \\
\text { At time } \tau^{n-1}: & A_{j(i, n+1)}^{n-1}=A_{j}^{n+1}+\frac{\left(S_{i}-A_{j}^{n+1}\right)\left(\tau^{n+1}-\tau^{n-1}\right)}{T-\tau^{n-1}},
\end{aligned}
$$

where $T \geq \tau^{n+1}>\tau^{n}>\tau^{n-1}$. It is interesting to observe that for the last step when $\tau^{n+1}=T$, equation (3.18) simplifies to $A_{j(i, n+1)}^{n}=A_{j(i, n+1)}^{n-1}=S_{i}$. The various quantities $(\cdot)_{i, j(i, n+1)}^{n}$ in equations (3.14-3.16) are determined by interpolation along lines of constant $S=S_{i}$. Assuming that the $S$ derivatives and the integral term are discretized using second order accurate methods, then it follows from [9] that at least quadratic interpolation should be used for $(\cdot)_{i, j(i, n+1)}^{n}$ in order to retain global second order convergence. 


\section{Semi-Lagrangian Timestepping and Discrete Observations}

It is common to consider continuously observed Asian options as the limit of discretely observed Asian options as the observation interval tends to zero $[15,37]$. In this section, we show that if the discrete sampling period is equal to the discrete PDE timestep, then a fully implicit, discretely sampled model is algebraically identical to a fully implicit semi-Lagrangian discretization of a continuously observed model. In the following, we ignore the effect of the boundary condition (2.17).

Consider the discrete average computed at discrete averaging times $t^{\ell}=\ell \Delta t$.

$$
A\left(t^{\ell}\right)=\frac{1}{\ell} \sum_{p=1}^{\ell} S\left(t^{p}\right)
$$

Equation (4.1) can be written

$$
A\left(t^{\ell+1}\right)=A\left(t^{\ell}\right)+\frac{S\left(t^{\ell+1}\right)-A\left(t^{\ell}\right)}{\ell+1}
$$

When using a PDE method to price a discretely observed Asian option, we consider that $V=V(S, A, t)$, and regard $(S, A)$ as independent variables. Suppose we have $N$ observation dates, at the times $\Delta t, 2 \Delta t, \ldots, N \Delta t$ with $N \Delta t=T$. Then at the $\ell$-th observation date we must have, by no arbitrage [39],

$$
\begin{aligned}
V\left(S, A\left(t^{\ell+1}\right), t^{(\ell+1)+}\right)= & V\left(S, A\left(t^{\ell}\right), t^{(\ell+1)-}\right) \\
& A\left(t^{\ell+1}\right)=A\left(t^{\ell}\right)+\frac{S-A\left(t^{\ell}\right)}{\ell+1}
\end{aligned}
$$

where $t^{(\ell+1)+}, t^{(\ell+1)-}$ are the instants just after and just before the observation date $t^{\ell+1}$. Note that $A\left(t^{\ell+1}\right)$ is regarded as constant for $t^{\ell+1}<t<t^{\ell+2}$. Let $\ell=N-k$, so that $k$ counts backwards. Since $\tau^{k}=k \Delta \tau$ we have that

$$
t^{\ell}=\ell \Delta t=(N-k) \Delta t=T-(k \Delta \tau)=T-\tau^{k}
$$

and similarly $t^{\ell+1}=T-\tau^{k-1}$. As well, we have that $t^{(\ell+1)+}=T-\tau^{(k-1)-}, t^{(\ell+1)-}=T-\tau^{(k-1)+}$. Writing the jump condition (4.3) in terms of the variable $\tau=T-t$ rather than $t$ then gives

$$
\begin{aligned}
V\left(S, A\left(\tau^{k+1}\right), \tau^{k+}\right)= & V\left(S, A\left(\tau^{k}\right), \tau^{k-}\right) \\
& A\left(\tau^{k}\right)=A\left(\tau^{k+1}\right)+\frac{S-A\left(\tau^{k+1}\right)}{N-k}
\end{aligned}
$$

Note that in this case we regard $A\left(\tau^{k+1}\right)$ as fixed during $\tau^{k}<\tau<\tau^{k+1}$.

Consider the case of a discretely observed European Asian option. In this case we solve

$$
V_{\tau}=C V+\mathcal{B} V+q(V)
$$

on the domain $\left[0, S_{\max }\right] \times\left[0, A_{\max }\right]$, with the jump conditions (4.5) imposed at observation times. Away from observation dates, if we discretize equation (4.6) in the $A$ direction then equation (4.6) represents a set of one dimensional PIDEs, which communicate only through jump conditions [15].

Set $A^{k}=A\left(\tau^{k}\right)$. Then we can write the jump condition (4.5) as

$$
\begin{aligned}
V\left(S, A^{k}, \tau^{k-}\right)= & V\left(S, A^{k+1}, \tau^{k+}\right) \\
& A^{k}=A^{k+1}+\frac{\left(S-A^{k+1}\right) \Delta \tau}{T-\tau^{k}} .
\end{aligned}
$$


Recalling that $A^{k+1}$ is constant during $\tau^{k+} \leq \tau \leq \tau^{(k+1)-}$, then a fully implicit discretizaton of equation (4.6) gives

$$
\frac{V\left(S_{i}, A_{j}^{k+1}, \tau^{(k+1)-}\right)-V\left(S_{i}, A_{j}^{k+1}, \tau^{k+}\right)}{\Delta \tau}=(C V)_{i, j}^{k+1}+(\mathcal{B} V)_{i, j}^{k+1}+q\left(V_{i, j}^{k+1}\right) .
$$

Note that this is a set of independent one dimensional PDEs (there are no $A$ derivatives in equation (4.8), $A_{j}^{k+1}$ appears only as a parameter). Using the jump condition (4.7) in equation (4.8) gives

$$
\begin{aligned}
\frac{V\left(S_{i}, A_{j}^{k+1}, \tau^{(k+1)-}\right)-V\left(S_{i}, A_{j(i, k+1)}^{k}, \tau^{k-}\right)}{\Delta \tau}= & (C V)_{i, j}^{k+1}+(\mathcal{B} V)_{i, j}^{k+1}+q\left(V_{i, j}^{k+1}\right) \\
& A_{j(i, k+1)}^{k}=A_{j}^{k+1}+\frac{\left(S-A_{j}^{k+1}\right) \Delta \tau}{T-\tau^{k}}
\end{aligned}
$$

which we recognize from equation (3.14) and equation (3.18) as being algebraically identical to a semiLagrangian, fully implicit discretization.

Note that in order for this result to hold, we must have discrete observations at $t=\Delta t, 2 \Delta t, \ldots, N \Delta t$, that is, no observation at $t=0$. Of course, in the limit as $\Delta t \rightarrow 0$, adding an extra observation at $t=0$ will be the same to $O(\Delta t)$ as the semi-Lagrangian solution.

Remark 4.1. As discussed in [22], it is straightforward to show that the common lattice methods used to price Asian options [24] are simply explicit finite difference methods for discretely observed models of Asian options. In many lattice applications, the observation interval is set to the lattice timestep, hence the continuously observed price is computed in the limit of vanishing timestep. A straightforward extension of the results above can be used to show that these lattice methods are simply explicit semi-Lagrangian methods. In this case, it is also easy to derive the conditions on the order of interpolation and the spacing on the lattice in the average direction to ensure optimal convergence. We note that, as discussed in [22], this is a point of confusion in the finance literature, and has led to schemes which are not, in fact, convergent $[22,6]$.

\section{Monotonicity and Stability of the Discrete Equations}

As shown in [34], in the case of nonlinear option pricing problems, seemingly reasonable discretization schemes can converge to an incorrect (i.e. non-viscosity) solution. Convergence to the viscosity solution is guaranteed if the discretization is consistent, monotone and $l_{\infty}$ stable $[5,12,13]$. Usually, consistency follows if any reasonable discretization method is used, although in the case of jump-diffusion, the non-locality of the integral term requires care in showing consistency $[12,13] . l_{\infty}$ stability is usually a consequence of monotonicity. Consequently, the most interesting requirement is monotonicity.

In the following, we will investigate the monotonicity and stability properties of the discrete equations. We will use a definition of monotonicity which is somewhat stricter than is usually the case in financial applications [5], but more in line with the definition used in computational fluid dynamics (CFD) [25]. It appears to us that the CFD definition is a more useful aid to the design of suitable discrete schemes. We will also indicate how these results can be extended to the case of more difficult nonlinear problems as in [34].

We remind the reader that use of semi-Lagrangian timestepping decouples the discrete equations at each timestep, resulting in a set of one dimensional discrete PIDEs. Hence we can use our techniques in $[21,34,16,17]$ to prove the desired properties of the discretized equations. 


\subsection{Preliminaries}

Define the matrices $B$ and $C$ such that

$$
\begin{aligned}
& {\left[B \cdot V_{j}^{n}\right]_{i}=(\mathcal{B V})_{i, j}^{n}+\text { truncation error }} \\
& {\left[C \cdot V_{j}^{n}\right]_{i}=(C V)_{i, j}^{n}+\text { truncation error }}
\end{aligned}
$$

where $V_{j}^{n}$ is the vector of discrete solution values $\left[V_{j}^{n}\right]_{i}=V\left(A_{j}, S_{i}, \tau^{n}\right)$ for fixed $A_{j}$. A detailed description of $B$ is given in [17, 16]. For our purposes, we note that $B$ has the properties given by equation (3.8). A detailed description of matrix $C$ is given in Appendix A.

To avoid algebraic complication, we will describe the discrete equations and the method used to solve the algebraic equations, only for the fully implicit and Crank-Nicolson timestepping methods. The reader should have no difficulty generalizing the results to the BDF case.

Let $\Phi^{n+1}$ be the Lagrange interpolation operator such that

$$
\left(\Phi^{n+1} \cdot V^{n}\right)_{i, j}=V\left(S_{i}, A_{j(i, n+1)}^{n}, \tau^{n}\right)+\text { interpolation error }
$$

where $\Phi^{n+1}$ is a linear operator for any order (linear, quadratic) of interpolation. We also let $V^{*}$ be the vector of payoffs obtained upon exercise and $P$ be the diagonal matrix given by

$$
P\left(V_{j}^{n+1}\right)_{i i}= \begin{cases}\text { Large } & \text { if } V_{i, j}^{n+1}<V_{i, j}^{*} \\ 0 & \text { otherwise. }\end{cases}
$$

Then the matrix form of the discrete equations for the penalized method is given by

$$
\begin{aligned}
{\left[I-(1-\theta) \Delta \tau C+P\left(V_{j}^{n+1}\right)\right] V_{j}^{n+1}=} & {\left[\Phi^{n+1}[I+\theta \Delta \tau C] V^{n}\right]_{j}+(1-\theta) \lambda \Delta \tau B V_{j}^{n+1} } \\
& +\left[\Phi^{n+1} \theta \lambda \Delta \tau B V^{n}\right]_{j}+\left[P\left(V_{j}^{n+1}\right)\right] V_{j}^{*}+\Delta \tau F_{j}^{n+1}
\end{aligned}
$$

for $j=1, .$. ,imax. Here $\theta=0$ is fully implicit, and $\theta=1 / 2$ is Crank-Nicolson timestepping. The term $F_{j}^{n+1}$ is used to approximate the boundary condition at $S=S_{\max }$, as discussed in subsection 2.2. Boundary condition (2.17) is enforced at $i=$ imax by setting

$$
B_{\text {imax }, l}=0
$$

for $l=1, \ldots$, imax, adjusting $C_{\text {imax }, l}$ as discussed in Appendix A, and letting

$$
\left[F_{j}^{n+1}\right]_{i}=\left\{\begin{array}{cl}
0 & , i \neq \operatorname{imax} \\
(1-\theta) \chi\left(S_{\max }, \tau^{n+1}\right)+\theta \chi\left(S_{\max }, \tau^{n}\right) & , i=\operatorname{imax}
\end{array}\right.
$$

where $\chi(S, \tau)$ is discussed in subsection 2.2.

Remark 5.1 (Viscosity Solution). In general, we may have non-smooth solutions to equation (2.5). This may be due to the degeneracy of the diffusion operator (no diffusion in the A direction), and due to possible non-smoothness in the payoff function. In these cases, we seek the viscosity solution to equation (2.5) [5]. Detailed discussions concerning existence and uniqueness of viscosity solutions to PIDEs in financial contexts are given in [31,2]. Sufficient conditions for convergence of a numerical scheme to the viscosity solution are given in [5]. A proof that the sufficient conditions in [5] (for PDEs) can be extended to nonlinear PIDEs is given in [12]. 
We note here that the penalty formulation of the American option pricing problem reduces problem (2.10) to the nonlinear PIDE (3.5), and hence the results in [12] apply. Briefly, if the numerical scheme is consistent, $l_{\infty}$ stable, and monotone, then convergence to the viscosity solution is guaranteed. In order to obtain a monotone scheme, we can use at most linear interpolation in equation (5.3).

The choice of interpolation scheme is discussed in [22] and [20]. Specifically, if the interpolation error does not get damped out, the global interpolation error after $N$ timesteps is $O\left(\frac{\left(\Delta S_{\max }\right)^{q}}{\Delta \tau}\right)$, where $q=2$ for linear interpolation, $q=3$ for quadratic interpolation and $\Delta S_{\max }=\max _{i}\left(S_{i+1}-S_{i}\right)$. Assuming second order in space and time truncation errors, the global discretization error is

$$
\text { global discretization error }=O\left[\frac{\left(\Delta S_{\max }\right)^{q}}{\Delta \tau}+\left(\Delta S_{\max }\right)^{2}+(\Delta \tau)^{2}\right],
$$

If we assume $\Delta S_{\max }=$ const.h and $\Delta \tau=$ const.h, then equation (5.9) reduces to

$$
\text { global discretization error }=O\left[\min \left(\left(h^{q-1}, h^{2}\right)\right]\right.
$$

\subsection{Monotonicity and Stability}

The highest order interpolation method $\Phi$ which will result in a monotone scheme is linear interpolation. Equation (5.10) suggests that if linear interpolation is used $(q=2)$, we can obtain no more than first order convergence. With this in mind, in the following analysis, we will consider only a fully implicit timestepping, and a linear interpolant $\Phi$ (as in equation (5.3)). We will, however, carry out numerical experiments with Crank-Nicolson and BDF timestepping, and higher order interpolants. The fully implicit version of equation (5.6) is

$$
\left[I-\Delta \tau C-\lambda \Delta \tau B+P\left(V_{j}^{n+1}\right)\right] V_{j}^{n+1}=\left[\Phi^{n+1} V^{n}\right]_{j}+P\left(V_{j}^{n+1}\right) V_{j}^{*}+\Delta \tau F_{j}^{n+1}
$$

for $j=1, \ldots$, imax.

As discussed in [5, 12], consistency, stability, and monotonicity are sufficient conditions for a numerical scheme to ensure convergence to the viscosity solution. In view of the importance of discretizations which are stable and monotone, both from a theoretical and practical point of view, it is useful to gather together a set of results for the implicit discretization schemes.

Lemma 5.1 (Properties of Matrix $C$ ). The matrix $C$ in equation (5.11) has the properties

$$
\begin{aligned}
\sum_{j} C_{i j} & =-(r+\lambda) ; i=1, \ldots, \text { imax }-1 \\
C_{i j} & \geq 0 ; i \neq j ; i=1, \ldots, \text { imax }-1 \\
C_{i j} & =0 ; j=1, \ldots, \text { imax } ; i=\operatorname{imax}
\end{aligned}
$$

Proof. This follows directly from Appendix A, and equation (5.7).

Set

$$
Q=I-\Delta \tau C-\lambda \Delta \tau B
$$

Lemma 5.2 (M-matrix property of $Q$ ). The matrix $Q$ is an $M$ matrix.

Proof. From equation (3.8) we have that $-B$ has non-positive offdiagonal elements. From Lemma 5.1, we have that $-C-\lambda B$ has non-positive offdiagonal elements. From Lemma 5.1, and properties (3.8), we have that

$$
\sum_{j}[-C-\lambda B]_{i j} \geq 0 ; i=1, \ldots, i \max
$$

and hence $Q$ is an $M$ matrix. 
We can write the discrete equations at each node $\left(S_{i}, A_{j}\right)$ as

$$
\begin{aligned}
g_{i, j}\left(V_{i, j}^{n+1},\left\{V_{k, j}^{n+1}\right\}_{i},\left\{V^{n}\right\}\right) & =-\left[Q V_{j}^{n+1}\right]_{i}+\left[\Phi^{n+1} V^{n}\right]_{i, j}+\left[P\left(V_{j}^{n+1}\right)\right]_{i i}\left(V_{i, j}^{*}-V_{i, j}^{n+1}\right)+\Delta \tau F_{i, j}^{n+1} \\
& =0
\end{aligned}
$$

where $\left\{V_{k, j}^{n+1}\right\}_{i}$ is to be interpreted as the set of values $V_{k, j}^{n+1}, k \neq i . k=1, \ldots$, imax, and $\left\{V^{n}\right\}$ is the set $V_{k, \ell}^{n}, k=1, . .$, imax $; \ell=1, \ldots$, imax.

Definition 5.1 (Monotone Discretizations). A discretization of the form (5.15) is monotone if

$$
\begin{aligned}
& g_{i, j}\left(V_{i, j}^{n+1},\left\{V_{k, j}^{n+1}+\rho_{k, j}^{n+1}\right\}_{i},\left\{V_{k, \ell}^{n}+\rho_{k, \ell}^{n}\right\}\right) \geq g_{i, j}\left(V_{i, j}^{n+1},\left\{V_{k, j}^{n+1}\right\}_{i},\left\{V^{n}\right\}\right) \quad \forall i, j ; \quad \forall k \neq i \\
& \forall \rho_{k, \ell}^{n} \geq 0, \forall \rho_{k, j}^{n+1} \geq 0, \\
& g_{i, j}\left(V_{i, j}^{n+1}+\rho_{i, j}^{n+1},\left\{V_{k, j}^{n+1}\right\}_{i},\left\{V^{n}\right\}\right)<g_{i, j}\left(V_{i, j}^{n+1},\left\{V_{k, j}^{n+1}\right\}_{i},\left\{V^{n}\right\}\right) \quad \forall i, j ; \quad \forall k \neq i \\
& \forall \rho_{i, j}^{n+1} \geq 0
\end{aligned}
$$

Remark 5.2. The above definition of monotonicity includes the condition (5.17). In the viscosity solution literature [5], only condition (5.16) is used to define monotonicity. However, in the conservation law literature [25, 23] monotonicity is usually defined including condition (5.17). In Appendix B, we outline the rationale for including condition (5.17). We believe that Definition 5.1 is a more useful definition of monotonicity.

Theorem 5.1 (Monotonicity of the Discretization). The fully implicit discretization (5.15) is unconditionally monotone.

Proof. We rewrite equation (5.15) as

$$
g_{i, j}=-\left[Q V_{j}^{n+1}\right]_{i}+\left[\Phi^{n+1} V^{n}\right]_{i, j}+\left[P\left(V_{j}^{n+1}\right)\right]_{i i}\left(V_{i, j}^{*}-V_{i, j}^{n+1}\right)+\Delta \tau F_{i, j}^{n+1}
$$

and examine each term in equation (5.18). From Lemma 5.2, matrix $Q$ is an $M$ matrix, hence $-\left[Q V_{j}^{n+1}\right]_{i}$ is a strictly decreasing function of $V_{i, j}^{n+1}$, and a non-decreasing function of $\left\{V_{k, j}^{n+1}\right\}_{i}$. Since $\Phi^{n+1}$ is a linear interpolant operator, $\left[\Phi^{n+1} V^{n}\right]_{i, j}$ is a non-decreasing function of $\left\{V^{n}\right\}$. Finally we see that the term $\left[P\left(V_{j}^{n+1}\right)\right]_{i i}\left(V_{i, j}^{*}-V_{i, j}^{n+1}\right)$ is a non-increasing function of $V_{i, j}^{n+1}$. Hence the discretization is monotone from Definition 5.1.

Theorem 5.2 (Stability of the Fully Implicit Scheme). The fully implicit method satisfies

$$
\left\|V^{n+1}\right\|_{\infty} \leq \max \left(\left\|V^{n}\right\|_{\infty},\left\|V^{*}\right\|_{\infty}\right)+\Delta \tau \chi_{\max }
$$

where $\chi\left(S_{\max }, \tau\right)$ is defined in subsection 2.2 , and

$$
\chi_{\max }=\max _{0 \leq \tau \leq T}\left|\chi\left(S_{\max }, \tau\right)\right| .
$$

In particular,

$$
\left\|V^{n+1}\right\|_{\infty} \leq\left\|V^{*}\right\|_{\infty}+T \chi_{\max }
$$

where $(n+1) \Delta \tau \leq T$. 
Proof. Writing out equation (5.11) in component form gives (see Appendix A)

$$
\begin{gathered}
V_{i, j}^{n+1}\left(1+\left(\alpha_{i}+\beta_{i}+r+\lambda\right) \Delta \tau\right)-\alpha_{i} \Delta \tau V_{i-1, j}^{n+1}-\beta_{i} \Delta \tau V_{i+1, j}^{n+1}-\lambda \Delta \tau \sum_{k} b_{i k} V_{k, j}^{n+1}+P_{i i}\left(V_{j}^{n+1}\right) V_{i, j}^{n+1} \\
=\sum_{k, l} w_{k, l}^{i, j} V_{k, l}^{n}+P_{i i}\left(V_{j}^{n+1}\right) V_{i, j}^{*}+\Delta \tau F_{i, j}^{n+1}
\end{gathered}
$$

where $w_{k, l}^{i, j}$ are linear interpolation weights satisfying

$$
0 \leq w_{k, l}^{i, j} \leq 1 \text { and } \sum_{k, l} w_{k, l}^{i, j}=1
$$

In addition, we recall that $B$ has properties (3.8)

$$
0 \leq b_{i k} \leq 1 \quad ; \quad \sum_{k} b_{i k} \leq 1
$$

while from Appendix A we have that

$$
\alpha_{i} \geq 0 \text { and } \beta_{i} \geq 0
$$

Let $m$ be an index such that

$$
\left|V_{m, j}^{n+1}\right|=\left\|V_{j}^{n+1}\right\|_{\infty} .
$$

Then equations (5.22-5.25) imply that

$$
\left\|V_{j}^{n+1}\right\|_{\infty}\left(1+r \Delta \tau+P\left(V_{j}^{n+1}\right)_{m m}\right) \leq\left\|V^{n}\right\|_{\infty}+P\left(V_{j}^{n+1}\right)_{m m}\left\|V^{*}\right\|_{\infty}+\Delta \tau \chi_{\max }
$$

and so

$$
\begin{aligned}
\left\|V_{j}^{n+1}\right\|_{\infty} & \leq \max \left(\left\|V^{n}\right\|_{\infty},\left\|V^{*}\right\|_{\infty}\right) \frac{1+P\left(V_{j}^{n+1}\right)_{m m}}{1+r \Delta \tau+P\left(V_{j}^{n+1}\right)_{m m}}+\frac{\Delta \tau \chi_{\max }}{1+r \Delta \tau+P\left(V_{j}^{n+1}\right)_{m m}} \\
& \leq \max \left(\left\|V^{n}\right\|_{\infty},\left\|V^{*}\right\|_{\infty}\right)+\Delta \tau \chi_{\max } .
\end{aligned}
$$

Hence

$$
\left\|V^{n+1}\right\|_{\infty} \leq \max \left(\left\|V^{n}\right\|_{\infty},\left\|V^{*}\right\|_{\infty}\right)+\Delta \tau \chi_{\max } .
$$

Therefore, by induction we have

$$
\left\|V^{n+1}\right\|_{\infty} \leq \max \left(\left\|V^{n-i}\right\|_{\infty},\left\|V^{*}\right\|_{\infty}\right)+(i+1) \Delta \tau \chi_{\max }
$$

for all $i$. Equation (5.21) follows from setting $i=n$ in equation (5.30).

Remark 5.3 (Extension to nonlinear models). It is completely straightforward to include a transaction cost or uncertain volatility model in the basic option pricing PIDE [33], which makes the PIDE nonlinear (even in the European case). For example, using the methods in [33], it is a simple exercise to extend the above stability and monotonicity results to the case of an American Asian option, with jumps and transaction costs. 


\subsection{Properties of a Semi-implicit Discretization}

Suppose we alter the discretization (5.11) so that the jump integral term is evaluated explicitly. Then

$$
\left[I-\Delta \tau C+P\left(V_{j}^{n+1}\right)\right] V_{j}^{n+1}=\lambda \Delta \tau B V_{j}^{n}+\left[\Phi^{n+1} V^{n}\right]_{j}+P\left(V_{j}^{n+1}\right) V_{j}^{*}+\Delta \tau F_{j}^{n+1}
$$

for $j=1, \ldots$, imax. The previous methods can also be applied to determine stability and monotonicity properties of this second discretization.

Theorem 5.3 (Stability and Monotonicity of Explicit Evaluation of the Jump Term). The discretization (5.31) is unconditionally stable and monotone.

Proof. Set $R=[I-\Delta \tau C]$. Then we can rewrite equation (5.31) as

$$
g_{i, j}=-\left[R V_{j}^{n+1}\right]_{i}+\left[\Phi^{n+1} V^{n}\right]_{i, j}+\left[P\left(V_{j}^{n+1}\right)\right]_{i i}\left(V_{i, j}^{*}-V_{i, j}^{n+1}\right)+\Delta \tau F_{i, j}^{n+1}+\left[\Delta \tau \lambda B V^{n}\right]_{i, j}
$$

From Lemma 5.1 and the properties of matrix $B$ (equation (3.8)), and following along the lines used to prove Theorem 5.1, it is straightforward to see that Definition 5.1 holds unconditionally for equation (5.32). Using a similar maximum analysis as in the proof of Theorem 5.2, we obtain unconditional stability.

Remark 5.4. Scheme (5.31) is very simple to implement, and retains unconditional monotonicity and stability. This method appears to have been completely overlooked. However, this scheme is only first order correct in time.

\section{Additional Properties of the Discrete Equations}

In this section we investigate how well our discrete approximation (3.5) preserves important properties of our original problem (2.10). We focus on two important properties : how well does the discrete penalty method satisfy the inequality constraints in problem (2.10) and does the discretization preserve arbitrage inequalities [13].

\subsection{Error in the Penalty Formulation}

In our original problem (2.10) we need to solve $\min \left(\mathcal{H} V ; V-V^{*}\right)=0$. In particular, we require that

$$
\left(V-V^{*}\right) \geq 0 \text {. }
$$

In discrete terms this becomes

$$
\left(V_{i, j}^{n+1}-V_{i, j}^{*}\right) \geq 0 .
$$

However, the penalty formulation (5.6) will result in $V_{i, j}^{n+1}<V_{i, j}^{*}$ at nodes in the exercise region. In this subsection we show that at these nodes we have $V_{i, j}^{n+1}=V_{i, j}^{*}-\varepsilon$, where $0<\varepsilon \ll 1$. In particular, we have the following bound on the error in the penalty term.

Lemma 6.1 (Error generated by the penalty formulation). Assume that $V^{*}$ satisfies a Lipschitz condition and suppose that

$$
\frac{\Delta \tau}{\Delta S_{\min }}<\text { const. as } \Delta \tau, \Delta S_{\min } \rightarrow 0
$$

where $\Delta S_{\min }=\min _{i}\left(S_{i+1}-S_{i}\right)$. Then

$$
V_{i, j}^{n+1}-V_{i, j}^{*} \geq-\frac{C_{1}}{\text { Large }}
$$

where $C_{1}$ is a positive constant independent of $\Delta S, \Delta \tau$. 
Proof. Let $k$ be an index such that

$$
\left(V_{k, j}^{*}-V_{k, j}^{n+1}\right)=\max _{i}\left(V_{i, j}^{*}-V_{i, j}\right) .
$$

Since the matrix $Q$ defined by (5.13) is an $M$ matrix from Lemma 5.2, it follows from equation (6.5) that

$$
\left[Q\left(V_{j}^{*}-V_{j}^{n+1}\right)\right]_{k} \geq 0
$$

and hence

$$
\left[Q V_{j}^{*}\right]_{k} \geq\left[Q V_{j}^{n+1}\right]_{k}
$$

From equation (5.18) we have that for all $j$

$$
Q V_{j}^{n+1}=\left[\Phi V^{n}\right]_{j}+P\left(V_{j}^{n+1}\right)\left(V_{j}^{*}-V_{j}^{n+1}\right)+\Delta \tau F_{j}^{n+1} .
$$

In particular, row $k$ of equation (6.8) is

$$
\left[Q V_{j}^{n+1}\right]_{k}=\left[\left[\Phi V^{n}\right]_{j}\right]_{k}+\left[P\left(V_{j}^{n+1}\right)\left(V_{j}^{*}-V_{j}^{n+1}\right)\right]_{k}+\Delta \tau F_{k, j}^{n+1} .
$$

Since

$$
\left[P\left(V_{j}^{n+1}\right)\left(V_{j}^{*}-V_{j}^{n+1}\right)\right]_{k}=\left\|P\left(V_{j}^{n+1}\right)\left(V_{j}^{*}-V_{j}^{n+1}\right)\right\|_{\infty}
$$

then equation (6.9) gives (using equation (6.7))

$$
\left\|P\left(V_{j}^{n+1}\right)\left(V_{j}^{*}-V_{j}^{n+1}\right)\right\|_{\infty} \leq\left\|V^{n}\right\|_{\infty}+\left\|Q V^{*}\right\|_{\infty}+\Delta \tau \chi_{\max } .
$$

From Theorem 5.2, and equation (6.11) we have

$$
\left\|P\left(V_{j}^{n+1}\right)\left(V_{j}^{*}-V_{j}^{n+1}\right)\right\|_{\infty} \leq C_{2}+\left\|Q V^{*}\right\|_{\infty},
$$

where $C_{2}=\left\|V^{*}\right\|_{\infty}+T \chi_{\max }$, with $(n+1) \Delta \tau \leq T$. Assuming that $V^{*}$ satisfies a Lipschitz condition, then

$$
\left\|Q V^{*}\right\|_{\infty} \leq C_{3} \frac{\Delta \tau}{\Delta S_{\min }}
$$

which follows from Lemma 5.2 and Appendix A. Assuming $\Delta \tau / \Delta S_{\min }$ is bounded, we have

$$
\left(V_{i, j}^{n+1}-V_{i, j}^{*}\right) \geq-\frac{C_{1}}{\text { Large }}
$$

with $C_{1}=C_{2}+\frac{C_{3} \Delta \tau}{\Delta S_{\min }}>0$.

Remark 6.1 (Significance of Lemma 6.1). Lemma 6.1 shows that the error induced by approximating problem (2.10) by the penalized system (3.6) can be made arbitrarily small by making Large (equation (5.4)) sufficiently large, provided the grid size is reduced such that $\Delta \tau / \Delta S_{\min }$ is bounded. In practice, this condition is not restrictive, since it does not make any sense to drive the spatial grid error to zero, leaving a finite timestepping error. Of course, when using finite precision arithmetic, we are limited to in practice due to the unit roundoff, which limits our ability to distinguish (numerically) $\left(V_{i, j}^{n+1}-V_{i, j}^{*}\right)$ from zero. As discussed in [21], this is not a problem of practical concern, since roundoff causes difficulty only when seeking to enforce condition (6.2) to unrealistic levels of accuracy. 
Theorem 6.1 (Discrete Comparison Principle). The fully implicit discretization (5.11) satisfies a discrete comparison principle, that is, if $V^{n}>W^{n}$ and $V^{n+1}, W^{n+1}$ satisfy equation (5.11), then $V^{n+1}>W^{n+1}$.

Proof. Suppose $V^{n}>W^{n}$. Write equation (5.11) for $V, W$

$$
\begin{aligned}
Q V_{j}^{n+1} & =\left[\Phi^{n+1} V^{n}\right]_{j}+\left[P\left(V_{j}^{n+1}\right)\right]\left(V_{j}^{*}-V_{j}^{n+1}\right)+\Delta \tau F_{j}^{n+1} \\
Q W_{j}^{n+1} & =\left[\Phi^{n+1} W^{n}\right]_{j}+\left[P\left(W_{j}^{n+1}\right)\right]\left(V_{j}^{*}-W_{j}^{n+1}\right)+\Delta \tau F_{j}^{n+1}
\end{aligned}
$$

Some manipulation of equation (6.15) results in

$$
\begin{aligned}
Q\left(V_{j}-W_{j}\right)^{n+1}= & -P\left(W_{j}^{n+1}\right)\left(V_{j}-W_{j}\right)^{n+1}+\left(P\left(V_{j}^{n+1}\right)-P\left(W_{j}^{n+1}\right)\left(V_{j}^{*}-V_{j}^{n+1}\right)\right. \\
& +\left[\Phi^{n+1}\left(V^{n}-W^{n}\right)\right]_{j}
\end{aligned}
$$

or

$$
\left[Q+P\left(W_{j}^{n+1}\right)\right]\left(V_{j}-W_{j}\right)^{n+1}=\left(P\left(V_{j}^{n+1}\right)-P\left(W_{j}^{n+1}\right)\left(V_{j}^{*}-V_{j}^{n+1}\right)+\left[\Phi^{n+1}\left(V^{n}-W^{n}\right)\right]_{j}\right.
$$

Since $Q$ is an $M$ matrix we have that $\left[Q+P\left(W_{j}^{n+1}\right)\right]$ is also an $M$ matrix. From equation (5.4), we have that

$$
\left(P\left(V_{j}^{n+1}\right)-P\left(W_{j}^{n+1}\right)\left(V_{j}^{*}-V_{j}^{n+1}\right) \geq 0\right.
$$

If linear interpolation is used, then $\left(V^{n}-W^{n}\right)>0$ implies that $\left[\Phi^{n+1}\left(V^{n}-W^{n}\right)\right]_{j}>0$. Finally, since $\left[Q+P\left(W_{j}^{n+1}\right)\right]$ is an $M$ matrix, its inverse satisfies $\left[Q+P\left(W_{j}^{n+1}\right)\right]^{-1} \geq 0$, and $\operatorname{diag}\left(\left[Q+P\left(W_{j}^{n+1}\right)\right]^{-1}\right)>0$, and hence $\left(V_{j}-W_{j}\right)^{n+1}>0$.

Remark 6.2. As discussed in [13], Lemma 6.1 has the financial interpretation that the discrete option prices satisfy arbitrage inequalities, that is, the inequality of payoffs is preserved in the inequalities of option prices.

\section{Iterative Solution of the Discretized Equations}

In order to solve equation (5.6), we use the following iteration scheme 


\section{Iteration}

For $j=1,2, \ldots$

Let $\left(V_{j}^{n+1}\right)^{0}=\left(V_{j}^{n}\right)$

Let $\hat{V}_{j}^{k}=\left(V_{j}^{n+1}\right)^{k}$

Let $\hat{P}^{k}=P\left(\left(V_{j}^{n+1}\right)^{k}\right)$

For $k=0,1,2, \ldots$ until convergence

Solve

$\left[I-(1-\theta) C+\hat{P}^{k}\right] \hat{V}_{j}^{k+1}$

$=\left[\Phi^{n+1}[I+\theta C] V^{n}\right]_{j}+\hat{P}^{k} V_{j}^{*}+\Delta \tau F_{j}^{n+1}$

$+(1-\theta) \lambda \Delta \tau B \hat{V}_{j}^{k}+\theta \lambda \Delta \tau\left[\Phi^{n+1} B V^{n}\right]_{j}$

If $\max _{i} \frac{\left|\hat{V}_{i, j}^{k+1}-\hat{V}_{i, j}^{k}\right|}{\max \left(1,\left|\hat{V}_{i, j}^{k+1}\right|\right)}<$ tol then break

EndFor

EndFor

For clarity, we have given algorithm 7.1 only for Crank-Nicolson and fully implicit timestepping. However, it is trivial to generalize this method to BDF timestepping. Note that each iteration of algorithm (7.1) requires a tridiagonal factor and solve, and a forward and back FFT (to evaluate $B \cdot \hat{V}_{j}^{k}$ ).

The following Theorem indicates that iteration scheme (7.1) is globally convergent.

Theorem 7.1 (Convergence of Iteration). Let matrices $C, B$ and $\hat{P}$ be given by (5.2), (5.1) and (5.4), respectively. Assume that matrix $I-(1-\theta) C$ is an M-matrix (which follows from Lemma 5.1), and that $B$ has properties (3.8). Then iteration (7.1) is globally convergent to the unique solution of equation (5.6) for any initial iterate $\hat{V}^{0}$.

Proof. Note that the algebraic equations (5.6) are decoupled for each line of constant $A_{j}$. Hence the issue of convergence of scheme (7.1) reduces to the convergence of each set of equations for constant $j$. But for constant $j$, this iteration is equivalent to solution of the discrete penalized equations for one dimensional American options with jump diffusion as described in [16]. Hence the result follows directly from Theorem 4.2 in [16].

\section{Computational Details and Numerical Results}

This section presents numerical results for various options and payoffs, including vanilla European call/put and American options. We will use an unequally spaced grid in the $A, S$ directions, on the domain $\left[0, S_{\max }\right] \times$ $\left[0, A_{\max }\right]$, with $A_{\max }=S_{\max }$. Probabilistic arguments can be used to determine an appropriate value for $S_{\max }$ [37]. We use $S_{\max }=50 K$, where $K$ is the strike. We describe below some tests which were carried out to verify that the effect of imposing boundary conditions at $S=50 \mathrm{~K}$ results in insignificant error. The convergence tolerance in iteration (7.1) was tol $=10^{-6}$. As suggested in [21], we choose Large $=1 /$ tol. If Crank-Nicolson or BDF timestepping is used, then quadratic interpolation is used in equation (5.3). If fully implicit timestepping is employed, then linear interpolation is used in equation (5.3). 
Given an $A$ grid discretization, the discrete PIDEs (3.14-3.15) become decoupled. At each timestep, we have a set of independent one dimensional discrete PIDEs to solve. This property makes solution of the continuously observed Asian option straightforward to implement, given an existing library which supports pricing of path dependent options.

As pointed out in equation (5.10), it is necessary to use at least a quadratic Lagrange interpolation scheme to find the solution at the foot of the characteristic curve, if we hope to obtain quadratic convergence. This will, however, result in a scheme which is not monotone.

The convergence ratio $R$ is defined in the following way. For each test, as we double the number of grid points in both $S$ and $A$ directions, we cut the timesteps $(\Delta \tau)$ in half. Let $\Delta \tau=\max _{n}\left(\tau^{n+1}-\tau^{n}\right), \Delta A_{\max }=$ $\max _{j}\left(A_{j+1}-A_{j}\right)$. Note that we are allowing here for the possibility of using variable timestep sizes (to be explained later), although most of our tests will simply use a constant timestep size. If we then carry out a convergence study, letting $h \rightarrow 0$, where $\Delta S_{\max }=$ Const. $h, \Delta A_{\max }=$ Const. $h$, and $\Delta \tau=$ Const. $h$. then we can assume that the error in the solution (at a given node) is

$$
V_{\text {approx }}(h)=V_{\text {exact }}+\text { Const. } h^{\xi} \text {. }
$$

The convergence ratio is then defined as

$$
R=\frac{V_{\text {approx }}(h / 2)-V_{\text {approx }}(h)}{V_{\text {approx }}(h / 4)-V_{\text {approx }}(h / 2)} .
$$

In the case of quadratic convergence $(\xi=2)$, then $R=4$, while for linear convergence $(\xi=1), R=2$.

Table 1 Value of a continuously observed fixed strike European Asian call option (no jumps) with constant timesteps. The input parameters are $\sigma=.1, r=.1, T=.25, \lambda=0$ and $K=100$. We compare the results given using the Večeř [38] one dimensional model, and the semi-Lagrangian method presented here. CrankNicolson timestepping was used.

semi-Lagrangian

Večeř 1-D PDE [38]

\begin{tabular}{cccc|cccc} 
Size of & No. of & \multicolumn{2}{c|}{$S=100$} & Size of & No. of & \multicolumn{2}{c}{$S=100$} \\
$S$ and $A$ grids & timesteps & Value & $R$ & $S$ grids & timesteps & Value & $R$ \\
\hline 51 & 25 & 1.857193 & n.a. & 51 & 25 & 1.839863 & n.a. \\
101 & 50 & 1.853254 & n.a. & 101 & 50 & 1.848642 & n.a \\
201 & 100 & 1.852120 & 3.475 & 201 & 100 & 1.850851 & 3.974 \\
401 & 200 & 1.851781 & 3.338 & 401 & 200 & 1.851407 & 3.979 \\
801 & 400 & 1.851660 & 2.815 & 801 & 400 & 1.851546 & 3.987
\end{tabular}

Table 1 shows results for a low volatility case, European Asian option (no jumps), using the semiLagrangian approach. In this special case, the two dimensional PDE can be reduced to one dimension [38], which we will refer to as the Večeř PDE [38] in the following. Results obtained by solving the Večeř PDE numerically are also given in Table 1.

In Table 1, we can see that the convergence ratio $R$ for the semi-Lagrangian method is not quadratic $(R \neq 4)$, while for the Večeř PDE [38] quadratic convergence is found. As discussed in [38], the Večeř PDE is not convection dominated, hence it is straightforward to obtain accurate numerical solutions. We remind the reader that this clever reduction to one dimension cannot be used for American options. The discontinuity present in the payoff greatly affects the convergence of the semi-Lagrangian method, since there is very little diffusion in the $A$ direction, and the non-smoothness in the payoff is not smoothed out during the solution 
Table 2 Value of a continuously observed fixed strike Asian call option (no jumps) at $S=K=100$, constant Crank-Nicolson timestepping. The input parameters are $\sigma=.1, \lambda=0, r=.1, T=.25, K=100$. Convergence ratios (8.1) are presented for different timestepping schemes. The right boundary of the space discretization $\left[0, S_{\max }\right]$ domain is truncated at different values.

\begin{tabular}{ccccc}
\hline & \multicolumn{2}{c}{$S_{\max }=5 \times K$} & \multicolumn{2}{c}{$S_{\max }=50 \times K$} \\
Timesteps & $S, A$ grid nodes & Value & $S, A$ grid nodes & Value \\
\hline 25 & 51 & 1.857193 & 54 & 1.857193 \\
50 & 101 & 1.853254 & 109 & 1.853254 \\
100 & 201 & 1.852120 & 217 & 1.852120 \\
200 & 401 & 1.851781 & 433 & 1.851781 \\
400 & 801 & 1.851660 & 865 & 1.851660 \\
& & & & \\
\hline
\end{tabular}

phase. Since we need to use quadratic interpolation in the $A$ direction in order to determine the values of the solution at the feet of the characteristic curves, the interpolation may be affected by the non-smooth payoff, and may lower the observed rate of convergence.

In order to test the effect of the boundary condition (2.17) at $S=S_{\max }$, we show results using two different values of $S_{\max }$ in Table 2. This table would seem to indicate that there is a negligible error for options of this maturity incurred setting $S_{\max }=50 \mathrm{~K}$, and all subsequent results will be reported imposing condition (2.17) at $S_{\max }=50 \mathrm{~K}$.

Figures 1 and 2 graphically present the solution $V$ and the first derivative of the solution with respect to the stock price $V_{S}$ when Crank-Nicolson is used. The plots are all smooth and do not exhibit any oscillations. While not shown here, $V_{S S}$ also did not show any oscillations.

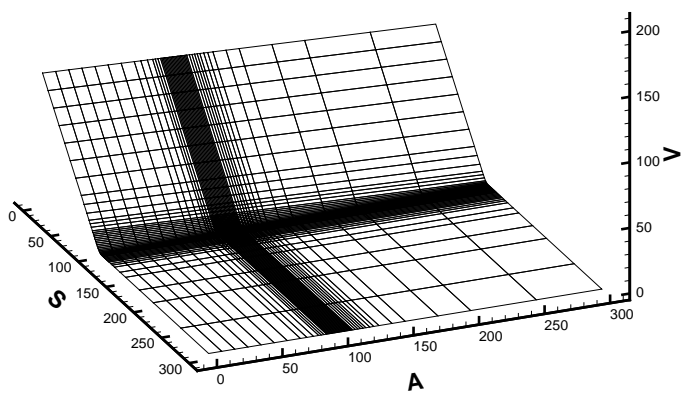

FIGURE 1: Value of a European fixed strike Asian put using Crank-Nicolson with constant timestepping $(\Delta \tau=.01) .51$ grid points are used both in the $A$ and $S$ direction. The input parameters are $\sigma=.1, r=.1, T=.25, K=100$, and $\lambda=0$.

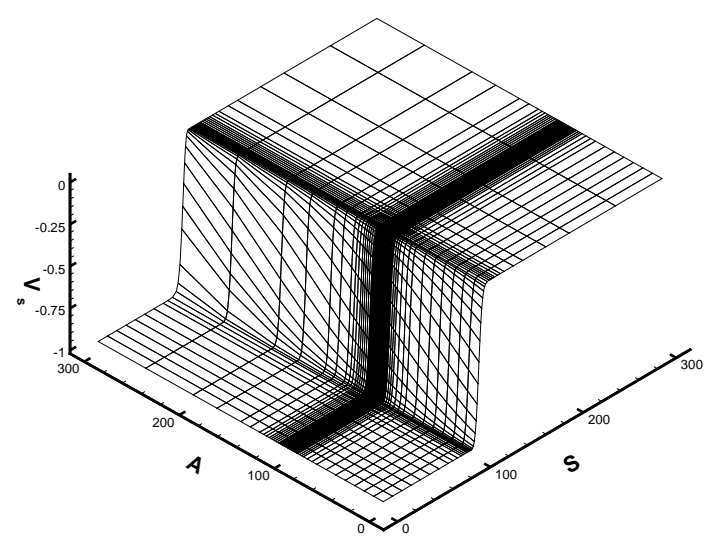

FIGURE 2: First derivative $\left(V_{S}\right)$ value of a European fixed strike Asian put using CrankNicolson with constant timestepping $(\Delta \tau=.01)$. 51 grid points are used both in the $A$ and $S$ direction. The input parameters are $\sigma=.1, r=.1$, $T=.25, K=100$ and $\lambda=0$.

We now explore numerical convergence for pricing Asian options for large values of volatility $\sigma$. Table 
3 presents our results. As expected quadratic convergence is recovered. In this case, a sufficient amount of diffusion in the $S$ direction appears to compensate for zero diffusion in the $A$ direction.

Table 3 Value of a continuously observed fixed strike Asian call (no jumps) option with constant timesteps at $S=k$. The input parameters are $\sigma=.5, r=.05, T=.25, \lambda=0$ and $K=100$. We compare the results given using the Večer 1-D PDE [38], and the semi-Lagrangian method presented here. Crank-Nicolson timestepping was used.

semi-Lagrangian

Večeř 1-D PDE [38]

\begin{tabular}{cccc|cccc} 
Size of & No. of & \multicolumn{2}{c|}{$S=100$} & Size of & No. of & \multicolumn{2}{c}{$S=100$} \\
$S$ and $A$ grids & timesteps & Value & $R$ & $S$ grids & timesteps & Value & $R$ \\
\hline 51 & 25 & 6.010203 & n.a. & 51 & 25 & 6.009821 & n.a. \\
101 & 50 & 6.015092 & n.a. & 101 & 50 & 6.014848 & n.a \\
201 & 100 & 6.016344 & 3.905 & 201 & 100 & 6.016251 & 3.582 \\
401 & 200 & 6.016651 & 4.085 & 401 & 200 & 6.016619 & 3.816 \\
801 & 400 & 6.016723 & 4.219 & 801 & 400 & 6.016713 & 3.915
\end{tabular}

\subsection{An In Depth Study of the Convergence Ratio}

The results of the previous section indicated that the semi-Lagrangian approach, coupled with CrankNicolson timestepping, results in quadratic convergence, for large volatilities. However, for small volatility values, quadratic convergence was not recovered. The goal of this subsection is to explore in detail different numerical techniques that could improve the convergence rate.

Table 4 Value of a continuously observed fixed strike Asian call option (no jumps) at the strike, constant timesteps. The input parameters are $\sigma=.1, r=.1, T=.25, \lambda=0, K=100$. Convergence ratios (8.1) are presented for different timestepping schemes: implicit, Crank-Nicolson and second order BDF.

\begin{tabular}{cccccccc}
\hline & & \multicolumn{3}{c}{ Implicit timestepping } & \multicolumn{2}{c}{ CN timestepping } & \multicolumn{2}{c}{ BDF timestepping } \\
Size of & No. of & \multicolumn{2}{c}{$S=100$} & \multicolumn{2}{c}{$S=100$} & \multicolumn{2}{c}{$S=100$} \\
$S$ and $A$ grids & timesteps & Value & $R$ & Value & $R$ & Value & $R$ \\
\hline 51 & 25 & 1.911865 & n.a. & 1.857193 & n.a & 1.86096 & n.a. \\
101 & 50 & 1.880801 & n.a. & 1.853254 & n.a & 1.854310 & n.a. \\
201 & 100 & 1.865907 & 2.086 & 1.852120 & 3.475 & 1.852416 & 3.513 \\
401 & 200 & 1.858681 & 2.061 & 1.851781 & 3.338 & 1.851868 & 3.453 \\
801 & 400 & 1.855112 & 2.025 & 1.851660 & 2.815 & 1.851686 & 3.014 \\
& & & & & & & \\
\hline
\end{tabular}

Table 4 contains the convergence rate results for different timestepping schemes for small volatility $(\sigma=.1$ and $r=.1)$. For implicit timestepping linear convergence is recovered $(R=2)$, as expected. However for higher order timestepping schemes such as Crank-Nicolson and second order backward differencing, quadratic convergence is not found (see Table 4). These results are not surprising since the combination of small volatility with the non-smooth payoff, means that quadratic interpolation in the $A$ direction is not $O\left(\left(\Delta A_{\max }\right)^{3}\right)$, for small $\tau$.

To try to remedy this problem, the initial payoff function is smoothed out. A classic method for handling discontinuities involves averaging the initial data. Specifically, values at each point are replaced with an 
average value over nearby space. Mathematically, we set

$$
\operatorname{PAYOFF}_{\text {smoothed }}\left(S_{i}, A_{j}\right)=\int_{K-\frac{\Delta A}{2}}^{K+\frac{\Delta A}{2}} \operatorname{PAYOFF}\left(S_{i}, A\right) d A \text {. }
$$

For a complete description of various smoothing methods the readers are referred to [33].

Table 5 Value of a continuously observed fixed strike call Asian call option (no jumps) at the strike with constant timesteps. The initial payoff is smoothed using the average scheme described by equation (8.2) The input parameters are $\sigma=.1, r=.1, T=.25, \lambda=0$, and $K=100$. Convergence ratios (8.1) are presented for different timestepping schemes: Crank-Nicolson and second order BDF.

\begin{tabular}{cccccc}
\hline & & \multicolumn{2}{c}{ CN timestepping } & \multicolumn{2}{c}{ BDF timestepping } \\
$\begin{array}{c}\text { Size of } \\
S \text { and } A \text { grids }\end{array}$ & $\begin{array}{c}\text { No. of } \\
\text { timesteps }\end{array}$ & Value & $R=100$ & Value & $R=100$ \\
\hline 51 & 25 & 1.870322 & n.a. & 1.874276 & n.a. \\
101 & 50 & 1.856377 & n.a. & 1.857462 & n.a. \\
201 & 100 & 1.852873 & 3.981 & 1.853179 & 3.925 \\
401 & 200 & 1.851963 & 3.849 & 1.852053 & 3.803 \\
801 & 400 & 1.851704 & 3.513 & 1.851731 & 3.497 \\
& & & & & \\
\hline
\end{tabular}

Table 5 contains the convergence rate results. From a convergence point of view, the ratios have improved in comparison with the convergence ratio without smoothing (see Table 4). However, quadratic convergence is still not obtained. From a theoretical point of view, all the convergence analysis for semiLagrangian scheme is based on the smooth properties of the solution $[9,20]$. If the solution is smooth then quadratic convergence is recovered. However, if the solution is non-smooth, then we can expect some reduction in the convergence rate.

Table 6 Value of a continuously observed Asian call option (no jumps) at the strike with constant timesteps. The input parameters are $\Delta \tau=.01, \sigma=.1, r=.1, T=.25, \lambda=0$ and $K=1$. Convergence ratios (8.1) are presented for the Crank-Nicolson timestepping scheme.

\begin{tabular}{cccc} 
Size of & No. of & \multicolumn{2}{c}{ Call option $\left.\left(\operatorname{PAYOFF}(A, K)=A^{2}-K\right)\right)$} \\
$S$ and $A$ grids & Value & $R=1$ \\
\hline 51 & 25 & 0.025749 & n.a. \\
101 & 50 & 0.025622 & n.a. \\
201 & 100 & 0.025591 & 4.131 \\
401 & 200 & 0.025584 & 4.093 \\
801 & 400 & 0.025582 & 4.054
\end{tabular}

To confirm our intuition that the non-smooth payoff is in fact the reason why quadratic convergence is not recovered, we create an artificial payoff that has the property of being quadratically smooth over the entire domain, e.g. $\operatorname{PAYOFF}(A, K)=\max \left(0, A^{2}-K\right)$. In this case quadratic convergence is recovered for both Crank-Nicolson and second order backward differencing. Table 6 shows detailed convergence results for Crank-Nicolson timestepping. 
Several other approaches were considered in an effort to improve convergence. We tried to use Rannacher timestepping [35]; two or more implicit timesteps are taken before reverting to a higher order timestepping scheme such as Crank-Nicolson for example. Numerical experiments indicated that this did not improve the convergence rate. A convergence rate of approximately 3.5 is found in this case. Adaptive timestepping was also considered [21] but this technique did not improve the convergence rate.

\subsection{Exotic Asian Options}

It is not generally possible to achieve second order convergence for American options using constant timesteps. In [21] it was demonstrated that in order to achieve second order convergence, it is necessary to use variable timestepping for American options. However, some initial tests showed that due to the large convective term in the $A$ direction, near $\tau=T$, the timestep selector suggested in [21] required very small timesteps near $\tau=T$. Consequently, we will show results in the following using constant timesteps.

Table 7 presents the input parameters. The mean of the jump distribution is denoted by $\mu$ and the jump distribution standard deviation is denoted by $\gamma$. These parameters are roughly the same as those estimated by [3] using European call options on the S\&P 500 stock index in April of 1999.

Table 7 Input data used to value American fixed strike Asian options under the lognormal jump diffusion process. These parameters are approximately the same as those reported in [3] using European call options on the S\&P 500 stock index in April of 1999.

\begin{tabular}{cccr}
\multicolumn{4}{c}{ Parameter values } \\
$\sigma$ & 0.15 & $\lambda$ & 0.10 \\
$r$ & 0.05 & $T$ & 0.25 \\
$\gamma$ & 0.45 & $K$ & 100.00 \\
$\mu$ & -0.90 & $\sigma_{\text {implied }}$ & 0.1886
\end{tabular}

To ensure consistent comparison between American Asian options with jumps and American Asian options without jumps, we proceed as follows:

1. Given the parameters in Table 7, we compute the analytical solution $V_{\text {jump }}$ at the strike $K$ of a vanilla put option, under jump diffusion.

2. Use a constant volatility Black-Scholes model with no jump to determine the implied volatility $\sigma_{\text {implied }}$ which matches the jump diffusion value $V_{\text {jump }}$ at the strike $K$.

3. Price the American Asian option with jumps using the parameters in Table 7.

4. Price the American Asian option with no jumps but with the implied volatility $\sigma_{\text {implied }}$ estimated in Step 2.

Table 8 compares the value of an American Asian fixed strike put option with the value of an American Asian fixed strike put option when the underlying stock follows the jump diffusion process described by [28]. Second order backward timestepping is used and the initial payoff is smoothed out using equation (8.2). We observe that quadratic convergence is not recovered, the convergence ratios are $\approx 3.5$. It is interesting to note that, at the strike, the price of an American Asian fixed strike put option with jumps is $9 \%$ cheaper than the price of the same option without jumps, while at $S=1.05 \mathrm{~K}$, the jump diffusion price is considerably higher than the no-jump price, as can be seen in Figure 3. 
Table 8 Value of a continuously observed fixed strike put American Asian option (under jump diffusion) with constant timestepping. Crank-Nicolson timestepping is used. The input parameters are defined in Table 7. This table presents convergence rates with and without jumps. Iterations refers to the total (over all timesteps) of the maximum number of iterations required for any value of $j$ (see algorithm 7.1) at each timestep.

\begin{tabular}{|c|c|c|c|c|c|c|c|}
\hline \multirow{3}{*}{$\begin{array}{c}\text { Size of } \\
S \text { and } A \text { grids }\end{array}$} & \multicolumn{4}{|c|}{ No jump } & \multicolumn{3}{|c|}{ Jumps } \\
\hline & \multirow{2}{*}{$\begin{array}{l}\text { No. of } \\
\text { timesteps }\end{array}$} & \multirow{2}{*}{$\begin{array}{l}\text { No. of } \\
\text { iterations }\end{array}$} & \multicolumn{2}{|c|}{$(S=100)$} & \multirow{2}{*}{$\begin{array}{l}\text { No. of } \\
\text { iterations }\end{array}$} & \multicolumn{2}{|c|}{$(S=100)$} \\
\hline & & & Value & $R$ & & Value & $R$ \\
\hline 51 & 25 & 77 & 2.220443 & n.a. & 99 & 2.044636 & n.a. \\
\hline 101 & 50 & 160 & 2.195726 & n.a. & 167 & 2.018530 & n.a \\
\hline 201 & 100 & 319 & 2.188555 & 3.447 & 340 & 2.012220 & 4.138 \\
\hline 401 & 200 & 692 & 2.186717 & 3.903 & 716 & 2.010691 & 4.126 \\
\hline 801 & 400 & 1397 & 2.186243 & 3.874 & 1609 & 2.010281 & 3.728 \\
\hline
\end{tabular}

Remark 8.1 (Alternative Boundary Condition). A simpler method of imposing boundary condition (2.17), is to simply set $V_{S S}=0$, and then discretize the $V_{S}$ term using one sided finite differences. As discussed in [40], this destroys the $M$ matrix property of the discretized equations $I-\Delta \tau C-\lambda \Delta \tau B$. Most of the theoretical results in this paper require that this $M$ matrix property hold, hence if we impose the boundary condition in this manner, these results cannot be proven to hold in this case. Nevertheless, we repeated all the computations reported above using this method of enforcing the boundary condition as $S \rightarrow \infty$. There was no change in the computed solution (at the strike) to eight digits.

\section{Conclusion}

In this paper we have put forward four primary contributions. First we have demonstrated that a semiLagrangian method can be used to price continuously observed American Asian options under jump diffusion processes. The implementation suggested here reduces this problem to solving a decoupled set of one dimensional discrete partial integral differential equations (PIDEs) at each timestep.

A second contribution is that since the discretized problem at each timestep reduces to a set of decoupled one dimensional PIDEs, we can make use of previous techniques developed by the authors to prove certain important properties of the discrete scheme, including convergence of the iterative method used to solve the implicit discrete equations.

In addition, we have included experimental computations which indicate that, even if second order timestepping methods are used, observed convergence as the mesh and timestep is refined occurs at a subsecond order rate. The problem can be traced to the non-smoothness of the payoff function.

Finally, we have also shown that in the fully implicit case, the semi-Lagrangian method for continuously observed Asian options is algebraically identical to a standard numerical method for pricing discretely observed Asian options, when the observation interval is equal to the discrete timestep. 


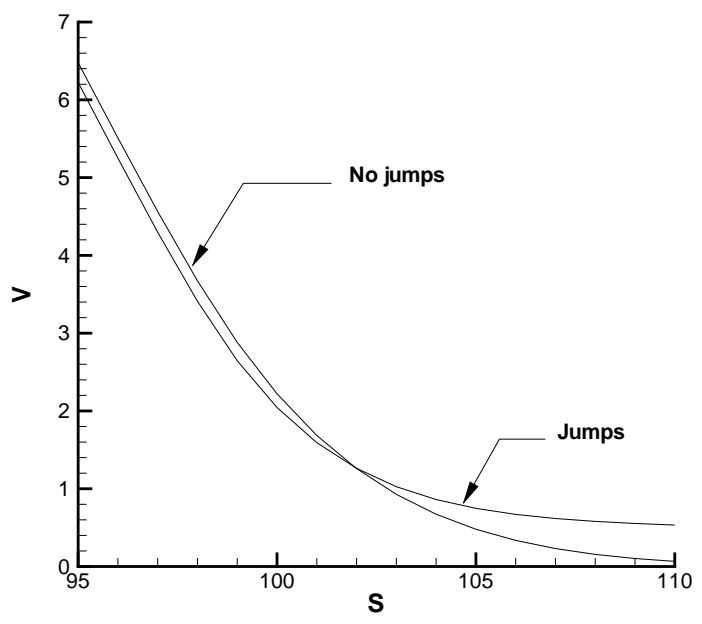

FIGURE 3: Comparison between the value of an American Asian fixed strike put option and the value of an American Asian fixed strike put option when the underlying stock follows the jump diffusion process described by [28]. The input parameters are defined in Table 7.

\section{Appendices}

\section{A Discretization of PDE}

In this appendix, we give the details of the discretization of the term $(C V)_{i, j}$ in equation (3.14).

Using finite differences, the matrix (5.2) is

$$
(C \cdot V)_{i}=-V_{i}^{n+1}\left(\alpha_{i}+\beta_{i}+r+\lambda\right)+\beta_{i} V_{i+1}^{n+1}+\alpha_{i} V_{i-1}^{n+1} ; i=2, \ldots, \text { imax }-1
$$

where $\alpha_{i}, \beta_{i}$ depend on the type of approximations used for the derivatives and second derivatives. For $i=1$, the matrix (A.1) reduces a diagonal entry with $\alpha_{1}, \beta_{1}=0$, and for the row $i=i m a x$, we set $\alpha_{i}=\beta_{i}=0$, and set $\lambda=0$.

There are a number of different discretizations of the derivative terms leading to various choices for $\alpha_{i}$ and $\beta_{i}$.

Discretizing the first derivative term of equation (3.2) with central differences leads to

$$
\begin{aligned}
\alpha_{i, c e n t r a l} & =\frac{\sigma_{i}^{2} S_{i}^{2}}{\left(S_{i}-S_{i-1}\right)\left(S_{i+1}-S_{i-1}\right)}-\frac{(r-\lambda \kappa) S_{i}}{S_{i+1}-S_{i-1}} \\
\beta_{i, \text { central }} & =\frac{\sigma_{i}^{2} S_{i}^{2}}{\left(S_{i+1}-S_{i}\right)\left(S_{i+1}-S_{i-1}\right)}+\frac{(r-\lambda \kappa) S_{i}}{S_{i+1}-S_{i-1}} .
\end{aligned}
$$

However if $\alpha_{i, c e n t r a l}$ or $\beta_{i, c e n t r a l}$ is negative, oscillations may appear in the solution. The oscillations can be avoided by using forward or backward differences at the problem nodes, leading to (forward difference)

$$
\begin{aligned}
\alpha_{i, f o r w a r d} & =\frac{\sigma_{i}^{2} S_{i}^{2}}{\left(S_{i}-S_{i-1}\right)\left(S_{i+1}-S_{i-1}\right)} \\
\beta_{i, f o r w a r d} & =\frac{\sigma_{i}^{2} S_{i}^{2}}{\left(S_{i+1}-S_{i}\right)\left(S_{i+1}-S_{i-1}\right)}+\frac{(r-\lambda \kappa) S_{i}}{S_{i+1}-S_{i}},
\end{aligned}
$$


or, (backward difference)

$$
\begin{aligned}
\alpha_{i, \text { backward }} & =\frac{\sigma_{i}^{2} S_{i}^{2}}{\left(S_{i}-S_{i-1}\right)\left(S_{i+1}-S_{i-1}\right)}-\frac{(r-\lambda \kappa) S_{i}}{S_{i+1}-S_{i}} \\
\beta_{i, \text { backward }} & =\frac{\sigma_{i}^{2} S_{i}^{2}}{\left(S_{i+1}-S_{i}\right)\left(S_{i+1}-S_{i-1}\right)} .
\end{aligned}
$$

Algorithmically, we decide between a central or forward discretization at each node for equation (A.1) as follows:

$$
\begin{aligned}
& \text { If }\left[\alpha_{i, \text { central }} \geq 0 \text { and } \beta_{i, \text { central }} \geq 0\right] \text { then } \\
& \quad \alpha_{i}=\alpha_{i, \text { central }} \\
& \quad \beta_{i}=\beta_{i, \text { central }} \\
& \text { ElseIf }\left[\beta_{i, \text { forward }} \geq 0\right] \text { then } \\
& \alpha_{i}=\alpha_{i, f o r w a r d} \\
& \beta_{i}=\beta_{i, \text { forward }}
\end{aligned}
$$

Else

$$
\begin{aligned}
& \alpha_{i}=\alpha_{i, \text { backward }} \\
& \beta_{i}=\beta_{i, \text { backward }}
\end{aligned}
$$

EndIf

Note that the test condition (A.5) guarantees that $\alpha_{i}$ and $\beta_{i}$ are non-negative. For typical values of $\sigma, r$ and grid spacing, forward differencing is rarely required for single factor options. In practice, since this occurs at only a small number of nodes remote from the region of interest, the limited use of a low order scheme does not result in poor convergence as the mesh is refined. For situations where the low order method causes excessive numerical diffusion, a flux limiter can be used [44]. As we shall see, requiring that all $\alpha_{i}$ and $\beta_{i}$ are non-negative has important theoretical ramifications. As $S \rightarrow 0$, equation (3.2) reduces to

$$
V_{\tau}=-r V
$$

which is simply incorporated into the discrete equations (A.1) by setting $\alpha_{i}, \beta_{i}, \lambda=0$ at $S_{i}=0$.

\section{B Practical Monotonicity}

If $g_{i j}$ in equation (5.15) is differentiable then we can restate Definition 5.1 as

$$
\begin{aligned}
\frac{\partial g_{i, j}}{\partial V_{i, j}^{n+1}} & <0 \\
\frac{\partial g_{i, j}}{\partial y} & \geq 0 ; y \in\left\{V_{k, j}^{n+1}\right\}_{i} \\
\frac{\partial g_{i, j}}{\partial z} & \geq 0 ; z \in\left\{V^{n}\right\}
\end{aligned}
$$


If $g_{i j}$ satisfies conditions (B.1), then we have immediately that

$$
\begin{aligned}
& \frac{\partial V_{i, j}^{n+1}}{\partial y} \geq 0 ; y \in\left\{V_{k, j}^{n+1}\right\} \\
& \frac{\partial V_{i, j}^{n+1}}{\partial z} \geq 0 ; z \in z \in\left\{V^{n}\right\}
\end{aligned}
$$

In computational fluid dynamics (CFD), condition (B.2) corresponds to requiring that discrete fluid flows mimic real flows (that is, increasing the pressure at neighbour nodes causes an increase in pressure in the central node). Hence Definition (5.1) is commonly used [25, 23].

Let

$$
\begin{aligned}
V_{\max }^{n} & =\max _{i j} V_{i, j}^{n} \\
V_{\text {min }}^{n} & =\min _{i j} V_{i, j}^{n} .
\end{aligned}
$$

Then, if $g_{i j}$ satisfies conditions (B.1) (or conditions (5.16-5.17)), we immediately have that

$$
\begin{array}{r}
g_{p, q}\left(V_{\text {max }}^{n+1}, V_{\text {max }}^{n+1}, V_{\text {max }}^{n}\right)=0 \\
g_{r, s}\left(V_{\text {min }}^{n+1}, V_{\text {min }}^{n+1}, V_{\text {min }}^{n}\right)=0
\end{array}
$$

where

$$
\begin{aligned}
V_{\max }^{n+1} & =V_{p, q}^{n+1} \\
V_{\min }^{n+1} & =V_{r, s}^{n+1} .
\end{aligned}
$$

Usually, it is easy to solve equation (B.4) for $V_{\max }^{n+1}, V_{\min }^{n+1}$, in terms of $V_{\max }^{n}, V_{\min }^{n}$, and hence determine if the discretization is $l_{\infty}$ stable.

In the finance literature, sufficient conditions for convergence to the viscosity solution are typically stated as monotonicity (condition (5.16)) and $l_{\infty}$ stability. Practically speaking, in order to determine if a scheme is $l_{\infty}$ stable, we will need condition (5.17) to hold as well as condition (5.16), hence we have defined monotonicity as requiring both conditions (5.16-5.17) consistent with the usage in the CFD literature.

\section{References}

[1] A. Allievi and R. Bermejo. Finite element modified method of characteristics for the Navier-Stokes equation. International Journal for Numerical Methods in Fluids, 32:439-464, 2000.

[2] A. L. Amadori. The obstacle problem for nonlinear integro-differential equations arising in option pricing. Working paper, Istituto pre le Applicazione del Calcolo "M. Picone", Rome, www.iac.rm.cnr.it $/$ amadori.

[3] L. Andersen and J. Andreasen. Jump-diffusion processes: Volatility smile fitting and numerical methods for option pricing. Review of Derivatives Research, 4:231-262, 2000.

[4] J. Andreasen. The pricing of discretely sampled Asian and lookback options. Journal of Computational Finance, 2:5-30, Fall 1998.

[5] G. Barles. Convergence of numerical schemes for degenerate parabolic equations arising in finance. In L. C. G. Rogers and D. Talay, editors, Numerical Methods in Finance, pages 1-21. Cambridge University Press, Cambridge, 1997. 
[6] J. Barraquand and T. Pudet. Pricing of American path-dependent contingent claims. Mathematical Finance, 6:17-51, 1996.

[7] D. S. Bates. Jumps and stochastic volatility: Exchange rate processes implicit in Deutsche mark options. Review of Financial Studies, 9:69-107, 1996.

[8] J. Becker. A second order backward difference method with variable timesteps for a parabolic problem. BIT - Numerical Mathematics, 38(4):644-662, 1998.

[9] R. Bermejo. Analysis of a class of quasi-monotone and conservative semi-Lagrangian advection schemes. Numerische Mathematik, 87:597-623, 2001.

[10] P. P. Boyle. New life forms on the option landscape. Journal of Financial Engineering, 2(3):217-252, 1996.

[11] P. P. Boyle and D. Emanuel. Options on the general mean. Working paper, University of British Columbia, 1980.

[12] M. Briani, C. La Chioma, and R. Natalini. Convergence of numerical schemes for viscosity solutions to integro-differential degenerate parabolic problems arising in financial theory. Working paper, Istituto pre le Applicazione del Calcolo "M. Picone", Rome, www.iac.rm.cnr.it/ $\sim$ natalini, To appear in Numerische Mathematik.

[13] R. Cont and E. Voltchkova. A finite difference scheme for option pricing in jump diffusion and exponential levy models. Internal Report 513, CMAP, Ecole Polytechnique, 2003.

[14] M.A.H. Dempster, J.P. Hutton, and D.G. Richards. LP valuation of exotic American options exploiting structure. Journal of Computational Finance, 2:61-84, Fall 1998.

[15] J.N. Dewynne and P. Wilmott. A note on average rate options with discrete sampling. SIAM Journal on Applied Mathematics, 55:267-277, 1995.

[16] Y. d'Halluin, P. A. Forsyth, and G. Labahn. A Penalty method for American options with jump diffusion processes. To appear in Numerische Mathematik, http://www.scicom.uwaterloo.ca/ $\sim$ ydhallui, 2004.

[17] Y. d'Halluin, P. A. Forsyth, and K. R. Vetzal. Robust numerical methods for contingent claims under jump diffusion processes. Submitted to IMA Journal of Numerical Analysis, http://www.scicom.uwaterloo.ca/paforsyt/jump.pdf, 2003.

[18] J. Douglas and T. F. Russel. Numerical methods for convection-dominated diffusion problems based on combining the method of characteritics with finite element or finite difference procedures. SIAM Journal on Numerical Analysis, 19:871-885, 1982.

[19] B. Eraker, M. Johannes, and N. Polson. The impact of jumps in volatility and returns. Journal of Finance, 58:1269-1300, 2003.

[20] M. Falcone and R. Ferretti. Convergence analysis for a class of high-order semi-Lagrangian advection schemes. SIAM Journal on Numerical Analysis, 35(3):909-940, 1998.

[21] P. A. Forsyth and K. R. Vetzal. Quadratic convergence of a penalty method for valuing American options. SIAM Journal on Scientific Computation, 23:2096-2123, 2002. 
[22] P. A. Forsyth, K. R. Vetzval, and R. Zvan. Convergence of numerical methods for valuing pathdependent option using interpolation. Review of Derivatives Research, 5:273-314, 2002.

[23] J. Fuhrmann and H. Langmach. Stability and existence of solutions of time-implicit finite volume schemes for viscous nonlinear conservation laws. Applied Numerical Mathematics, 37:201-230, 2001.

[24] J. Hull and A. White. Efficient procedures for pricing European and American path-dependent options. Journal of Derivatives, 1 (Fall):21-31, 1993.

[25] R.J. LeVeque. Numerical Methods for Conservation Laws. Birkhauser, 1992. Lectures in Mathematics.

[26] A. L. Lewis. Fear of jumps. Wilmott Magazine, December 2002.

[27] M. Marcozzi. On the valuation of Asian options by variational methods. SIAM Journal on Scientific Computing, 24:1124-1140, 2003.

[28] R. C. Merton. Option pricing when underlying stock returns are discontinuous. Journal of Financial Engineering, 3:125-144, 1976.

[29] G. H. Meyer. On pricing American and Asian options with PDE methods. Acta Mathematica Universitatis Comenianae, 70:153-165, 2001.

[30] K. Parrott and N. Clarke. A parallel solution of early exercise Asian options with stochastic volatility. 1998. Proceedings of the 11th Domain Decomposition Conference, Greenwich.

[31] H. Pham. Optimal stopping of controlled jump diffusion processes: a viscosity solution approach. Journal of Mathematical Systems, Estimation and Control, 8:1-27, 1998.

[32] O. Pironneau. On the transport diffusion algorithm and its applications to the Navier-Stokes equations. Numerische Mathematik, 38:309-332, 1982.

[33] D. M. Pooley, P. A. Forsyth, and K. R. Vetzal. Convergence remedies for non-smooth payoffs in option pricing. Journal of Computational Finance, 6(4):25-40, 2003.

[34] D.M. Pooley, P.A. Forsyth, and K.R. Vetzal. Numerical convergence properties of option pricing PDEs with uncertain volatility. IMA Journal of Numerical Analysis, 23:241-267, 2003.

[35] R. Rannacher. Finite element solution of diffusion problems with irregular data. Numerische Mathematik, 43:309-327, 1984.

[36] P. Ritchken and R. Trevor. Interest rate options with a volatility hump. Review of Derivatives Research, 3:237-262, 1999.

[37] D. Tavella and C. Randall. Pricing Financial Instruments: The Finite Difference Method. John Wiley \& Sons, Inc, 2000.

[38] J. Večeř. A new PDE approach for pricing arithmetic average Asian options. Journal of Computational Finance, 4:105-113, Summer 2001.

[39] P. Wilmott. Derivatives: The Theory and Practice of Financial Engineering. John Wiley \& Sons Ltd., West Sussex, England, 1998.

[40] H. Windcliff, P.A. Forsyth, and K.R. Vetzal. Analysis of the stability of the linear boundary condition for the Black-Scholes equation. 2003. To appear in Journal of Computational Finance. 
[41] R. Zvan, P. A. Forsyth, and K. R. Vetzal. Robust numerical methods for PDE models of Asian options. Journal of Computational Finance, 1 (Winter):39-78, 1998.

[42] R. Zvan, P. A. Forsyth, and K. R. Vetzal. Discrete Asian barrier options. Journal of Computational Finance, 3:41-67, 1999.

[43] R. Zvan, P.A. Forsyth, and K.R. Vetzal. Penalty methods for American options with stochastic volatility. Journal of Computational and Applied Mathematics, 91:199-218, 1998.

[44] R. Zvan, P.A. Forsyth, and K.R. Vetzal. A finite volume approach for contingent claims valuation. IMA Journal of Numerical Analysis, 21:703-731, 2001. 